Usage, Usability, and Utility of 3D City Models, 03002 (2012)

DOI: $10.1051 / 3 \mathrm{u} 3 \mathrm{~d} / 201203002$

(C) Owned by the authors, published by EDP Sciences, 2012

\title{
A new concept of perceptual fidelity to augment virtual urban environments with microclimatic ambiances
}

\author{
T. Vigier ${ }^{1}$, G. Moreau ${ }^{1}$, D. Siret ${ }^{1}$ and L. Lescop ${ }^{2}$ \\ ${ }^{1}$ LUNAM Université, CERMA UMR CNRS 1563, Nantes, France \\ ${ }^{2}$ École Nationale Supérieure d'Architecture de Nantes, GERSA, Nantes, France
}

\begin{abstract}
Whereas virtual reality has proven some potential for visual urban landscape assessment, we raise similar questions about multisensory evaluation. Urban perception is complex and putting aside sensory interactions biases the evaluation of future projects. We attempt more particularly to propose several solutions to integrate microclimatic ambiances in virtual environment evaluations. These propositions rely on a new concept of realism: perceptual fidelity. It consists in provoking the same perceptual effect as in reality without try to reproduce physical causes.
\end{abstract}

\section{INTRODUCTION}

Computer visualizations and 3D models are nowadays completely integrated into the design process and for urban landscape assessment. Moreover, virtual reality (VR) [1] has been proved to be a good tool to represent the urban environment. Thanks to immersion and interaction, users can navigate in a virtual environment where they feel present and on which they can act.

However, these tools are very often focused on visuals and only a few take into account dynamical and sensorial experience in cities. We focus more particularly on microclimatic ambiances which represent climatic factors (light, temperature, wind, humidity...) modified by the configuration of the built city.

From a review of the usage of VR for urban landscape and ambiances assessment, we raise the issue of sensory perceptions and microclimatic representations in VR modeling. Then we develop the concept of perceptual fidelity based on the notion of effect. From this concept, we propose different solutions to suggest microclimatic and multisensory urban ambiances only with an audio-visual environment.

\section{RELATED WORKS AND PROBLEMATIC}

Bishop ([2, 3]) is one of the firsts to use computer animations and VR to communicate about urban landscape. Digital images and photomontages were already used in previous works but they were often limited to only one aspect of the environment and could not tackle the movement function in urban perception. Indeed perception is very dependent on observer's action ([4]) and several previous works have highlighted the influence of walking in the evaluation of cities $([5,6])$.

According to Bishop et al. ([2]), VR can be used to communicate qualities of urban design, to interact with design features and make experimentations about human perception. In these first works, Bishop et al. have already shown numerous issues about realism in the design of virtual worlds for the urban environment assessment.

VR is also used to study urban ambiances and landscape ambiances. Thus, virtual evaluation focuses on something else than the visual aesthetics of buildings or the spatial configuration of the area. Tahrani

This is an Open Access article distributed under the terms of the Creative Commons Attribution License 2.0, which permits unrestricted use, distribution, and reproduction in any medium, provided the original work is properly cited. 
\& Moreau ([7]) propose a virtual evaluation of light in an urban walkthrough. However, as in the work of Bishop et al. perceptual biases are also problematic because they partly alter the assessment. These biases are due to less subtle contrasts of light than reality and the lack of characters and sounds. Furthermore by assessing a virtual wind turbines landscape, Jallouli \& Moreau ([8]) report that the lack of wind noise and skin sensation is mentioned by $30 \%$ of respondents.

These works show the relevance of the usage of VR for urban landscape assessments. VR is a proven solution to show on-going constructed projects, to visualize design choices and to escape from time and location. Moreover it permits to really experience a place thanks to immersion and interaction. In a political context where public participation is more and more present, VR makes it possible a non-expert representation of urban projects.

However in these examples the perception in cities is often oversimplified. Indeed, even if sounds have been added in some applications, evaluations are often based only on visuals to the expense of other sensory effects. Moreover complex perceptual phenomena as sensory interactions, anticipation and memorization are laid aside. This issue has been also developed by Lange ([9]) in a recent paper in which he reviews the advances in landscape visualization since the 1970's and evokes the lacks and the potential advances in this domain. According to him, "focusing only on the visual aspect of landscape design provides us with only a partial "view", literally, of our environment." He also underlines the lack of knowledge in the representation of a multisensory environment and "how such representations might influence landscape assessments, or how they could influence decision-making in planning and designing our environment".

In a multisensory evaluation of urban environments, microclimatic ambiances are significant ([10]). Microclimatic ambiances depend on the building configuration therefore they are localized and change all along an urban walkthrough. These transitory effects bring into play all senses and are very seldom integrated in virtual evaluation. Except in Tahrani \& Moreau's work ([7]), changes of the environmental ambiances are not modeled. Urban ambiances are unstable and physiological and psychological adaptations are essential in urban experience whether it is pleasant or not.

Beyond local transitions, we can notice that VR applications often simulate urban environments in ideal situations: the sky is blue, there are a few white clouds, vegetation is lush, trees are leafy or flowered, sun raises and the sun is shinny. Even if Bishop \& Rohrmann have simulated an environment by night ([3]) and Jallouli \& Moreau have added grey clouds to be closer to the real experience ([8]), participants can never experiment various seasons, times of day or weathers and compare and evaluate the urban design under different climate and lighting situations.

Some researchers have tried to represent dynamic and sensory features of urban ambiances but they have quite often deviated to scientific visualizations while they aimed at communicating to non-expert people. Onoue et al. ([11]) have tried to create a low-cost VR system (VRATE System, Figure 1) which provides heat and wind sensations. Hence, they use an avatar that users can identify with. The avatar is surrounded by a "mesh" containing thermal information and other information is displayed on screen. Unfortunately too much information is displayed and the interface is overloaded: the evaluation remains for experts and non-sensory.

In this paper, we attempt to determine how microclimatic ambiances could be integrated in virtual urban environments for non-expert evaluation.

\section{THE CONCEPT OF PERCEPTUAL FIDELITY}

\subsection{The possibility of sensory reproduction?}

The most intuitive, yet difficult to achieve, approach consists in reproducing the physical stimuli of microclimatic ambiances. For suggesting wind in VR, Moon \& Kim ([12]) propose a device named WindCube made up of small electric fans attached to a cubic structure. 


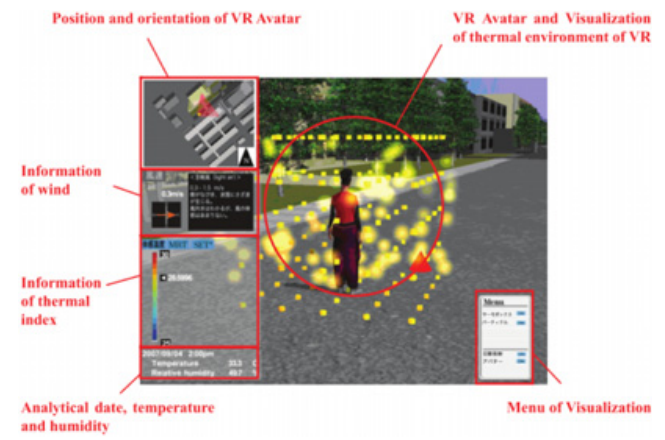

Figure 1. The interface design of VRATE System ([11]).

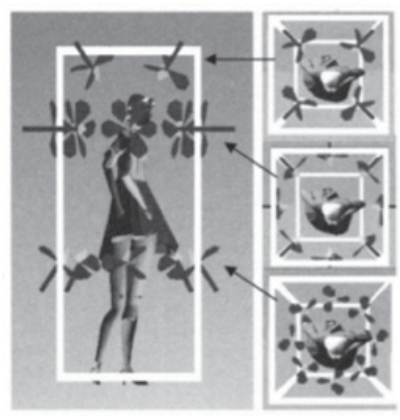

Figure 2. The placement of the fans in the WindCube ([12]).

For their part, Kulkarni et al. ([13, 14]) propose a system for wind simulation, "Treadport Active Wind Tunnel", based on computational fluid dynamics and able to simulate urban wind very precisely. This device also includes radiant panels to suggest heat. Besides, the representation of sunshine could be improved by "virtual shadows" ([15]). It consists in throwing shadows of virtual objects onto the floor. This technique is only adapted for immersive screen as CAVE or cylindrical screen.

However, these devices are just prototypes. The evaluation of the WindCube is really simplistic and we cannot find an experimental evaluation with human users in the Treadport Active Wind Tunnel. Moreover they are not easily accessible and are too often expensive. And because of fans' noise, visual pollution of devices, biases from reality or latency, they can have a negative effect. They can also be difficult to transport and to set up and become more of a constraint than a help to public participation.

A sensory reproduction could be envisaged but this solution entails too much technical constraints. We now propose another solution based on the concept of perceptual fidelity to suggest microclimatic and multisensory ambiances.

\subsection{Perceptual fidelity}

\subsubsection{The notion of effect}

The effect is a notion used in the field of architectural and urban ambiances in particular for "sonic effects" (or sound effects). Noticing that the concepts of "sound object" and the "soundscape" are respectively "too elementary (in terms of levels of organization)" and "too broad and blurred" for the analysis of the urban environment, Augoyard \& Torgue ([16]) define sonic effects as an intermediate notion between objectivity and subjectivity, between the cause and the event. "The concept of the sonic 
Usage, Usability, and Utility of 3D City Models

effect seemed to describe this interaction between physical sound environment, the sound milieu of a socio-cultural community, and the "internal soundscape" of every individual."

We think that this notion can be useful to study microclimatic ambiances in VR. Faced with technical limitations, it seems more interesting to suggest the effect on the person using only audiovisual interfaces rather than duplicating the natural sensory stimuli. Attention is paid to perceptual, cognitive or emotional responses. The notion of effect applied to virtual environments brings a new concept of realism: perceptual fidelity.

\subsubsection{The concept of perceptual fidelity}

Perceptual fidelity is reached when the virtual environment provokes the same perceptual responses as reality using the complexity of the perceptual process (sensory interactions, cognitive and memory processes, anticipation phenomena...). This concept is between the "there-reality" and the "feelrealism" ([17]).

According to Chalmers \& Ferko "there-reality environments are defined as those virtual environments which evoke the same perceptual response from a viewer as if they were actually present". This definition is based on the "perception equation":

$$
P(t, \rho)=\omega_{V} V+\omega_{A} A+\omega_{S} S+\omega_{T} T+\omega_{F} F
$$

Where $t$ is the task, $\rho$ is the preconditioning, $V=$ visuals, $A=$ audio, $S=$ smell, $T=$ taste and $F=$ feel. $\Sigma \omega_{I}=1$ and each $\omega_{I}$ represents the relative weight of each sense: it is the threshold such as above it there is no difference of perception with reality. Even if Chalmers \& Ferko consider sensory interactions by telling that "the effects of one sense may indeed alter the weighting of another" and by integrating preconditioning as a parameter of the response, they focus more on sensory stimuli and they do not consider the problems of sensory consistency.

On the other hand, Morie et al. ([18]) propose a higher-level concept: the "feel-realism". The goal is to reach a high degree of presence thanks to an emotional equivalence in the virtual world and the real world. Nevertheless, sensitive user responses are restricted to emotions. Perceptual fidelity extends these definitions of all perceptual responses (feeling, sensory stimulation, impression...) thanks to the notion of effect. The design of the virtual world is therefore $1 /$ the definition of all effects that users will experience and $2 /$ the implementation of solutions to provoke them. These effects could be unimodal, multisensory, broad, located, long, brief, transitory, etc. The solutions can be sensory reproductions and also metaphors, sensory interactions, cognitive suggestions, sensory substitutions, etc.

We are now going to apply perceptual fidelity to the suggestion of microclimatic ambiances in VR.

\section{PROPOSITIONS FOR THE SUGGESTION OF MICROCLIMATIC AMBIANCES IN VR}

In previous parts, we showed that we could use cognitive perception knowledge as sensory interactions, substitutions or metaphors to reach perceptual fidelity to suggest microclimatic effects in audiovisual virtual applications. Concerning thermal perception it is influenced by colors, materials, visual discomfort, light contrasts and cognitive expectation or preconditioning ([19]). Moreover wind sensation is often associated with temperature.

Artistic practices (paintings, cinema, comic strips, video games... ) are particularly efficient to propose visual and sound effects enabled to represent and suggest complex sensations, perceptions or emotions. For instance the colors and light works of impressionists to represent seasons (Figure 3), the shape or perspective deformations to suggest uneasiness (Figure 4). From the theoretical knowledge about perception and artistic know-how we are going to propose different solutions to create various microclimatic effects in VR. 

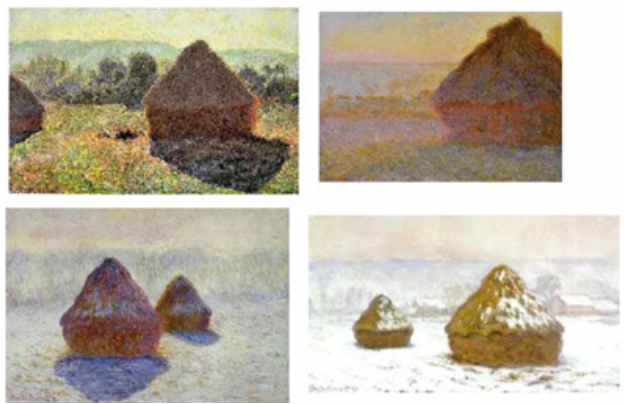

Figure 3. The ricks of Monet, 1890-1891 (spring, summer, autumn and winter).

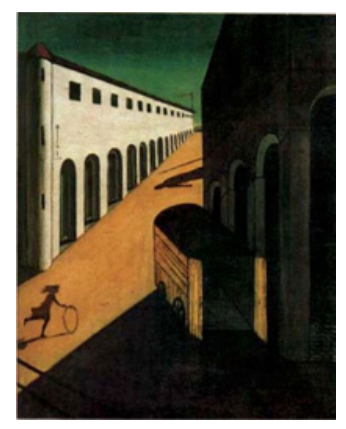

Figure 4. Mystère et mélancolie d'une rue, Chirico, 1914.

\subsection{Preconditioning and contextualization}

Human perception depends on environmental parameters but also on culture, previous experience, physical state and state of mind. Moreover thermal and wind effects depend on global weather (e.g. a gust is not felt in the same way in summer or winter).

It is thus necessary to have means to condition the psychological state of the participant and the context of the virtual experience. Preconditioning can be made by watching an explicative video ([18]). For thermal sensations preconditioning can be made with neutral transition spaces (e.g. white room, temperature around $20^{\circ} \mathrm{C}$, no furniture...). In urban assessment, preconditioning is particularly important to cut participants off from real place (city), season and time of day. Thus, a special care must be provided to clearly establish a climatic ambiance (light, colors, precipitations, global wind with cloud movements. ..) while designing the virtual world.

\subsection{Audio-visual propositions}

We develop in the following table (Table 1) some solutions to suggest microclimatic ambiances in an audio-visual environmewnt (the list is not exhaustive). Each effect is evaluated in terms of technical feasibility (repeatability, real-time solutions...).

Furthermore each solution distorts the basic 3D model to add microclimatic effects: a compromise must be found to suggest the intended effect without completely corrupting the perception of the scene. The goal is to bring a sensory and sensitive evaluation; effects must be therefore subtle and not surprising and nor too simplistic. For instance about color processing, we cannot use a simple red filter to suggest heat (or a blue one for cold) because the evaluation would become only cognitive and not sensitive. Another perceptual issue is the wind direction. Indeed the wind sensation highly depends 
Usage, Usability, and Utility of 3D City Models

Table 1. Audio-visual propositions to suggest microclimatic effects.

\begin{tabular}{|c|c|c|}
\hline Effect & Solution & Technical comments \\
\hline \multirow[t]{3}{*}{$\begin{array}{l}\text { Thermal effects due to } \\
\text { building shape }\end{array}$} & $\begin{array}{l}\text { Distortion of buildings (e.g. } \\
\text { narrowing to suggest heat; } \\
\text { opening to suggest freshness) }\end{array}$ & $\begin{array}{l}\text { How to put forward automatic } \\
\text { and real-time distortions that } \\
\text { are perceptually valid? }\end{array}$ \\
\hline & $\begin{array}{l}\text { Modification of colors either by } \\
\text { altering the light spectrum or by } \\
\text { using filters as in the painting of } \\
\text { Anquetin (Figure 5) }\end{array}$ & $\begin{array}{l}\text { Is color processing possible } \\
\text { with photo-textures? }\end{array}$ \\
\hline & $\begin{array}{l}\text { Adding a sound reverberation } \\
\text { filter to suggest heat trapping }\end{array}$ & \\
\hline \multirow[t]{3}{*}{$\begin{array}{l}\text { Sunshine transitions due } \\
\text { to building shape }\end{array}$} & $\begin{array}{l}\text { Enhancement of visual } \\
\text { accommodation (e.g. glare effect) }\end{array}$ & $\begin{array}{l}\text { Results will heavily depend on } \\
\text { the visual interface. }\end{array}$ \\
\hline & Emphasizing contrasts & $\begin{array}{l}\text { Results will heavily depend on } \\
\text { the visual interface. }\end{array}$ \\
\hline & Distortions of shadows & $\begin{array}{l}\text { How to put forward automatic } \\
\text { and real-time distortions that } \\
\text { are perceptually valid? }\end{array}$ \\
\hline $\begin{array}{l}\text { Sunshine effects due to } \\
\text { building and ground } \\
\text { materials }\end{array}$ & $\begin{array}{l}\text { Enhancing light scattering and } \\
\text { reflections }\end{array}$ & $\begin{array}{l}\text { We cannot use photo-textures. } \\
\text { Results will heavily depend on } \\
\text { the visual interface. }\end{array}$ \\
\hline \multirow{3}{*}{$\begin{array}{l}\text { Wind effect due to } \\
\text { building shape (e.g. } \\
\text { (corner effect, roll vortex } \\
\text { at the foot of a building, } \\
\text { venturi effect) }\end{array}$} & $\begin{array}{l}\text { Visual effects: leaves or flags } \\
\text { waving, particles }\end{array}$ & \\
\hline & $\begin{array}{l}\text { Sound effects: sound in trees, } \\
\text { whistling in ears }\end{array}$ & \\
\hline & $\begin{array}{l}\text { Using Beaufort scale to associate } \\
\text { wind speed with visual and } \\
\text { sensory effects (Figure 6) }\end{array}$ & \\
\hline
\end{tabular}

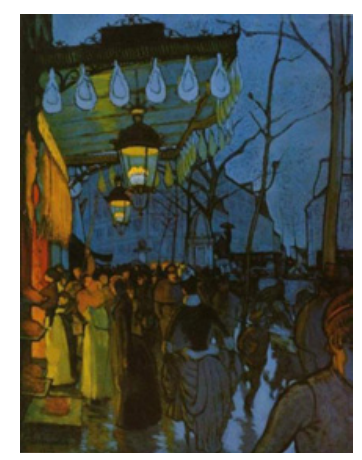

Figure 5. Avenue de Clichy, soir 5 heures, Anquetin, 1887. The painter observes the scene through a blue tinted glass to render the atmosphere of an autumn evening in Paris ([20]).

on its direction: a wind coming from behind would be perceived much less annoying and thus present than the same wind from the front.

A more detailed perceptual evaluation is to be done in a future work after a real experimentation. 


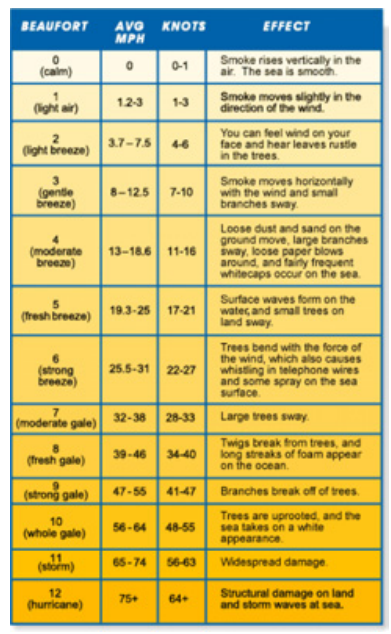

Figure 6. The Beaufort scale (source: http://www.horizonwind.com).

\subsection{Calibration and evaluation}

We have presented some solutions to suggest microclimatic effects in VR. These solutions should be calibrated before being used in virtual environments. Since the goal is creating the same perceptual responses than reality, calibrations should be qualitative and not necessary based on quantitative value of physical phenomena.

We chose two existing places in Nantes (France) to test these virtual effects. Evaluation should be done under various climatic and seasonal conditions because each effect should be differently implemented according to the context. Our future work will consist in:

- Specifying and characterizing which effects we want to suggest in these areas.

- Modeling and rendering these areas.

- Implementing the chosen effects from previous propositions in the $3 \mathrm{D}$ virtual environments according to the perceptual fidelity.

- Evaluating the virtual microclimatic effects in these environments. We could compare effects with footage and we should propose adequate methods of investigation.

- Propose some tools to enable planners to easily add sensory qualities in the project presentation or evaluation to general public.

\section{CONCLUSIONS}

In this paper, we have highlighted a new problematic about the usage of VR in urban landscape assessment: the recognition of complex and multisensory features of cities in particular microclimatic ambiances to transcend visual aesthetics. Thus we propose different audio-visual solutions to suggest microclimatic effects in low-cost and easily implementable way. These solutions are based on a new concept, perceptual fidelity, which consists in suggesting various defined perceptual effects without necessarily trying to reproduce original physical phenomena.

\section{References}

[1] P. Fuchs, G. Moreau, and P. Guitton, Virtual Reality: Concepts and Technologies. CRC Press (2011). 
[2] I. Bishop, W. Ye, and C. Karadaglis, "Experiential approaches to perception response in virtual worlds," Landscape and Urban Planning, 54 (2000)

[3] I. Bishop and B. Rohrmann, "Subjective responses to simulated and real environments: a comparison," Landscape and Urban Planning, 65 (2003)

[4] J. J. Gibson, The ecological approach to visual perception (1986)

[5] K. Lynch, The image of the city (1960)

[6] C. Gordon, The Concise Townscape (1976)

[7] S. Tahrani and G. Moreau, "Integration of Immersive Walking to Analyse Urban Daylighting Ambiences," Journal of Urban Design, 13 (2008)

[8] J. Jallouli and G. Moreau, "Virtual Reality as a Landscape Decision-Making Tool: the Wind Turbines' Case," International Journal of Design Sciences \& Technology, 16 (2010)

[9] E. Lange, "99 volumes later: We can visualise. Now what?," Landscape and Urban Planning, 100 (2011)

[10] M. Nikolopoulou and K. Steemers, "Thermal comfort and psychological adaptation as a guide for designing urban spaces," Energy and Buildings, 35 (2003)

[11] T. Onoue, N. Yabuki, S. Yoshida, and T. Fukuda, "Visualization technique of outdoor thermal environment using a VR avatar," in CONVR (2010)

[12] T. Moon and G. J. Kim, "Design and Evaluation of a Wind Display for Virtual Reality," in ACM VRST Conference (2004)

[13] S. D. Kulkarni, M. A. Minor, M. W. Deaver, and E. R. Pardyjak, "Output Feedback Control of Wind Display in a Virtual Environment," in IEEE/ICRA (2007)

[14] S. D. Kulkarni, M. A. Minor, E. R. Pardyjak, and J. M. Hollerbach, "Combined Wind Speed and Angle Control in a Virtual Environment Using a Static Observer," in IEEE/RSJ (2008)

[15] T. Naemura, T. Nitta, A. Mimura, and H. Harashima, "Virtual shadows in mixed reality environment using flashlight-like devices," Trans Virtual Reality Society of Japan, 7 (2002)

[16] J.-F. Augoyard and H. Torgue, Sonic experience: A guide to everyday sounds (2006)

[17] A. Chalmers and A. Ferko, "Levels of Realism: From Virtual Reality to Real Virtuality," in Spring Conference on Computer Graphics (2008)

[18] J. F. Morie, J. Williams, D.-P. Luigi, and A. Dozois, “The Fidelity of 'Feel': Emotional Affordance in Virtual Environments The role of emotions within VEs," in HCI International (2005)

[19] S. Narçon, "Caractérisation des perceptions thermiques en régime transitoire. Contribution à l'étude de l'influence des interactions sensorielles sur le confort", PhD Thesis, École Pratique des Hautes Études, France (2001)

[20] J.-L. Chalumeau, La ville dans l'art (2000) 